\title{
A combination of oral L-citrulline and L-arginine improved 10-min full-power cycling test performance in male collegiate soccer players: a randomized crossover trial
}

\author{
Izumi Suzuki $^{1} \cdot$ Keishoku Sakuraba $^{1} \cdot$ Takumi Horiike $^{1} \cdot$ Takafumi Kishi $^{1} \cdot$ Junya Yabe $^{1} \cdot$ Takashi Suzuki $^{2}$. \\ Masahiko Morita ${ }^{2} \cdot$ Akihito Nishimura $^{2} \cdot$ Yoshio Suzuki $^{1}$
}

Received: 30 June 2018 / Accepted: 6 February 2019 / Published online: 16 February 2019

(C) The Author(s) 2019

\begin{abstract}
Purpose Oral L-citrulline (Cit) increases plasma L-arginine (Arg) concentration and the production of nitric oxide (NO). NO dilates blood vessels and potentially improves sports performance. The combination of oral Arg and Cit (Arg + Cit) immediately and synergistically increases plasma Arg and nitrite/nitrate (NOx) concentrations more than either Cit or Arg alone. This prompted us to assess the effects of oral Arg + Cit on 10-min cycling performance in a double-blind, randomized, placebo-controlled crossover trial.

Methods Twenty-four male soccer players ingested either Cit + Arg or placebo (both $1.2 \mathrm{~g} /$ day each) for 6 days. On day 7, they ingested Cit + Arg $1 \mathrm{~h}$ before performing a 10-min full-power pedaling test on a bicycle ergometer. Plasma NOx and amino acid levels were measured before and after the test, as well as the participants' subjective perception of physical exertion.

Results Power output was significantly greater with Cit + Arg than in the placebo group ( $242 \pm 24$ vs. $231 \pm 21 \mathrm{~W} ; p<0.05)$. Plasma concentrations of post-exercise NOx $(p<0.05)$, Cit $(p<0.01)$ and Arg $(p<0.01)$ were significantly higher in the $\mathrm{Cit}+\mathrm{Arg}$ than in the placebo group, whereas exercise upregulated plasma NOx concentrations in both groups $(p<0.05)$. $\mathrm{Cit}+\mathrm{Arg}$ also gave improved post-exercise subjective perception of "leg muscle soreness" and "ease of pedaling" (both $p<0.05)$.

Conclusion Seven days of oral Citrulline $(1.2 \mathrm{~g} / \mathrm{d})$ and Arginine $(1.2 \mathrm{~g} / \mathrm{d})$ ingestion improved 10-min cycling performance and the perception of physical exertion in male collegiate soccer players.
\end{abstract}

Keywords Supplement $\cdot$ Pre-workout $\cdot$ Vasodilator $\cdot$ Ergogenic $\cdot$ Bicycle ergometry

\section{Abbreviations}

Arg: $\quad$ L-arginine

ASL: Argininosuccinate lyase

ASS1: Argininosuccinate synthase

BCAA: Branched chain amino acids

Cit: $\quad$ L-citrulline

NO: $\quad$ Nitric oxide

NOx: Nitrite/nitrate

Communicated by Anni Vanhatalo.

Yoshio Suzuki

yssuzuki@juntendo.ac.jp

1 Faculty of Health and Sports Science, Juntendo University, 1-1, Hiragagakuendai, Inzai, Chiba 270-1695, Japan

2 Research and Innovation Center, Kyowa Hakko Bio Co., Ltd, Miyukigaoka, Tsukuba, Ibaraki 305-0841, Japan
$\mathrm{NO}_{2}: \quad$ Nitrite

$\mathrm{NO}_{3}^{-}: \quad$ Nitrate

SEM: $\quad$ Standard error of the mean

VAS: Visual analogue scale

\section{Introduction}

Nitric oxide (NO) dilates blood vessels, enhancing circulation and improving mitochondrial efficiency (Albrecht et al. 2003; Tschakovsky and Joyner 2008; Larsen et al. 2011). A previous study suggests that NO positively regulates mitochondrial biogenesis through the upregulation of peroxisome proliferator-activated receptor- $\gamma$ co-activator- $1 \alpha($ PGC- $1 \alpha)$, a central regulator of mitochondrial function, because mitochondrial respiration and body energy balance are markedly abolished in eNOS-deficient animals (Nisoli et al. 2003). In 
addition, NO exerts a range of physiological functions [e.g., enhancing muscle contractile efficiency, improving exercise tolerance, and regulating oxygen consumption (Shen et al. 1994; Larsen et al. 2007; Bailey et al. 2010; Petróczi and Naughton 2010; Lansley et al. 2011a; Bescós et al. 2012; Jones et al. 2013)] acting as an intercellular messenger as well as forming reactive nitrogen species (Quijano et al. 2016). Some researchers have focused on the beneficial effects that dietary nitrate $\left(\mathrm{NO}_{3}{ }^{-}\right)$-containing supplements, such as beetroot juice, have on exercise performance (Bailey et al. 2009; Lansley et al. 2011b; Cermak et al. 2012a; Wilkerson et al. 2012; Jones 2014). However, other reports show no improvement in endurance performance after $\mathrm{NO}_{3}{ }^{-}$supplementation in highly trained elite athletes (Cermak et al. 2012b; Boorsma et al. 2014). Therefore, efforts have been directed towards strategies that promote NO production.

The endogenous synthesis of NO proceeds via the metabolism of L-arginine (Arg) to L-citrulline (Cit) by NO synthase (Curis et al. 2005). Argininosuccinate synthase (ASS1) and argininosuccinate lyase (ASL) then recycle Cit to Arg, which results in a Cit-Arg cycle that efficiently produces NO (Curis et al. 2005).

Arg is a conditionally essential amino acid that exerts various physiological actions by improving vascular endothelial (Bode-Boger et al. 2003; Lin et al. 2008; Siasos et al. 2008), physical (Campbell et al. 2006; Fricke et al. 2008; Koppo et al. 2009), and sexual (Chen et al. 1999) functions. These functions require high doses of Arg, since intestine degrades substantial amount ( 40\%) of dietary Arg and the remainder is taken up by the liver and metabolized to urea (Castillo et al. 1993a, b; Wu 1998; Van De Poll et al. 2004).

Cit is an $\alpha$-amino acid that is abundant in watermelon (Citrullus vulgaris) and is a potent endogenous precursor of Arg. Notably, oral Cit ingestion increases blood Arg concentrations more efficiently than an equal amount of Arg in humans (Schwedhelm et al. 2008). Cit is not metabolized in the small intestine or liver (Windmueller and Spaeth 1981; Van De Poll et al. 2007), which accounts for why oral Cit elevates Arg levels more effectively than Arg. We have recently demonstrated that Cit supplementation exhibits several beneficial effects on the cardiovascular system and endothelial function by enhancing NO production (Ochiai et al. 2012; Yabuki et al. 2013). A growing body of evidence also indicates that exercise performance is enhanced via NO production when healthy adults orally consume Cit (Bailey et al. 2015; Suzuki et al. 2016). Therefore, Cit could have physiological properties that efficiently elevate Arg and NO levels involving the modulation of exercise capacity.

Previous studies in animal models have shown that a combination of oral Cit and Arg increases blood Arg concentration immediately and more effectively than either Cit or Arg alone (Morita et al. 2014). Particularly in healthy humans, combined supplementation with Cit and Arg has been proven to exhibit acute elevation of plasma Arg levels within $1 \mathrm{~h}$ following intake: it then immediately demonstrates a half-life, $\mathrm{t}_{1 / 2}$, of 1.5-2 h (Suzuki et al. 2017). And in our previous study using the $4 \mathrm{~km}$ cycling time trial test $1 \mathrm{~h}$ after Cit supplementation (Suzuki et al. 2016), the mean completion time in Cit was significantly shorter than placebo (placebo: $578 \pm 15 \mathrm{~s}$, Cit: $569 \pm 14 \mathrm{~s}, p<0.05$ ). Therefore, we hypothesized that a $10 \mathrm{~min}(600 \mathrm{~s})$ full-power pedaling test at $1 \mathrm{~h}$ after intake, could detect the acute effects of oral Cit and Arg.

Previous studies (Bailey et al. 2015; Suzuki et al. 2016) have indicated that about 1 week of continuous Cit ingestion improves exercise performance, whereas a single bolus does not (Hickner et al. 2006; Cutrufello et al. 2015). The lowest dose and duration of Cit ingestion that is reportedly required to improve exercise performance is $2.4 \mathrm{~g} /$ day for 8 days (Suzuki et al. 2016). In addition, we found oral Cit and Arg combination synergistically increases plasma Arg levels compared with its ingestion of either alone (Morita et al. 2014; Suzuki et al. 2017). Therefore, we hypothesized that the Cit + Arg could exert the same effect at a lower dose of Cit. Here, we investigated the effects of $1.2 \mathrm{~g} /$ day each of oral Cit combined with Arg for 7 days on exercise performance.

\section{Materials and methods}

\section{Participants}

Twenty-four male soccer players aged 18 to 25 years volunteered to participate in this double-blind, randomized, placebo-controlled, two-arm crossover trial. All were members of Juntendo University's Division 1 soccer team, which is part of the Kanto University Football League. The purpose, methods, potential results, and review of the trial protocol, as well as the protection of personal information, potential benefits, and disadvantages of participating in the trial were explained to each participant. All the players understood that participation depended on their own free will and that they could withdraw at any time. All players then provided written, informed consent to participate in the trial. The participants were habitually using the ergometer thereby expected to be familiarized enough to perform 10 min pedaling test. After the screening PWC test, they were instructed to practice the 10 min pedaling test at their prescribed load prior to the 1 st trial so that to eliminate a learning effect.

The inclusion criteria were as follows: male collegiate soccer players, physical work capacity (PWC) ${ }_{75 \% \mathrm{HRmax}}>160 \mathrm{~W}$, and ability to pedal a bicycle ergometer at $>60 \mathrm{rpm}$ for 12 min with a workload set at their $\mathrm{PWC}_{75 \% \mathrm{HRmax}}$ at $60 \mathrm{rpm}$.

The exclusion criteria were as follows: goal keepers, under medical treatment, presently with, or having a history 
of cardiovascular, respiratory endocrine or metabolic disorders, liver or kidney dysfunction, chest pain, fainting, allergies to components related to the test supplement, taking supplements that include Cit or Arg, smoking, and seasonal allergies such as Cryptomeria japonica or Chamaecyparis obtusa pollenosis, blood donations of $>200 \mathrm{~mL}$, or of $400 \mathrm{~mL}$ within 1 or 3 months, respectively, before the bicycle ergometer test, participation in other clinical trials currently or within the past 3 months and being judged inappropriate by the study director.

The participants were instructed not to change their routine exercise habits and meal content during the study period.

The protocol was implemented according to the Declaration of Helsinki and was approved by the Ethics Committee of Juntendo University Graduate School of Sports and Health Sciences (Approval \#27-108) as well as the Research Ethics Review Board of Kyowa Hakko Kirin Co., Ltd. (Approval \#2015_043).

This study is registered at the UMIN clinical trial registry (UMIN-CTR; ID UMIN000021525).

Four participants were excluded from the analysis due to upper respiratory tract infections on the test day $(n=3)$ and missing data $(n=1)$. Data were analyzed from the remaining 20 participants (mean \pm SE; age, $19.0 \pm 0.2$ years; weight, $65.4 \pm 0.1 \mathrm{~kg}$; height, $173.1 \pm 1.1 \mathrm{~cm}$; body mass index, $\left.21.8 \pm 0.2 \mathrm{~kg} / \mathrm{m}^{2}\right)$.

\section{Physical work capacity tests}

We measured $\mathrm{PWC}_{75 \% \text { HRmax }}$ as described by Miyashita et al. (Miyashita et al. 1985) using a PowerMax VIII bicycle ergometer, (Konami, Tokyo, Japan), with three stages of load $(25,75$ and $125 \mathrm{~W})$ for $3 \mathrm{~min}$ each (total, $9 \mathrm{~min}$ ), and heart rate was measured using a heart rate monitor (RS $800 \mathrm{CX}$, Polar Japan, Tokyo, Japan). We calculated $\mathrm{PWC}_{75 \%}$ HRmax using a simple regression line derived from average heart rates for $30 \mathrm{~s}$ during the latter half of each stage and load intensity. Maximum heart rate was set at 220-age.

\section{Study design}

The participants executed one trial with a placebo and another with Cit + Arg after a washout period of 2 months. The order of the trials was randomized so as a half of the participants to take Cit + Arg first and the others to take placebo first using research randomizer (https://www.rando mizer.org/) in order to minimize the order effect.

The participants ingested the placebo or Cit + Arg for 7 days. Both were provided as granulated powders in a stick packet containing $2.4 \mathrm{~g}$ of maltitol (Mitsubishi Shoji Foodtech Co., Tokyo, Japan) or $1.2 \mathrm{~g}$ each of Cit and Arg (both Kyowa Hakko Bio Co., Ltd., Tokyo, Japan). Maltitol was used as a placebo because it has very similar appearance to Cit and Arg and no influences on our target outcomes to be evaluated. The appearance, weight, smell and taste of the Cit + Arg and placebo powders were confirmed to be indistinguishable by the manufacturer (Kyowa Hakko Bio Co., Ltd) of the test supplements and the practitioners prior to conduct this study.

Blood was collected from the cubital vein at the laboratory after an overnight fast on day 1 , and then the supplement was ingested with $200 \mathrm{~mL}$ of water. Between days 2 and $6, \mathrm{Arg}+\mathrm{Cit}$ or the placebo were ingested before training or at bedtime on non-training days.

During days 1-6, the time of supplement ingestion and physical and health status were diarized. After 15:00 on day 6 , the participants ingested only water, consumed a defined meal $(1500 \mathrm{kcal}$, protein $49.1 \mathrm{~g}$, lipid $63.1 \mathrm{~g}$, carbohydrate $182.9 \mathrm{~g}$ ) by $21: 00$ and then fasted.

The height, weight, blood pressure, heart rate, and selfreported physical and health status of the participants were evaluated at the laboratory, and blood was collected at 08:00 on day 7. The participants then consumed a rice ball (180 kcal, protein $3.6 \mathrm{~g}$, lipid $0.5 \mathrm{~g}$, carbohydrate $40.2 \mathrm{~g}$ ) and water (ad libitum up to $500 \mathrm{ml}$ ) and rested for $60 \mathrm{~min}$. The participants then ingested Cit + Arg or the placebo and rested for $35 \mathrm{~min}$, warmed up for $15 \mathrm{~min}$, rested for another $10 \mathrm{~min}$ (total of $60 \mathrm{~min}$ ) and then started the 10-min pedaling test. This sequence was based on a report showing that plasma Arg levels peak at $1 \mathrm{~h}$ after supplementation (Suzuki et al. 2017).

The 10-min pedaling test was conducted using a PowerMax VIII bicycle ergometer (Konami, Tokyo, Japan), with the torque set to output individual $\mathrm{PWC}_{75 \% \mathrm{HRmax}}$ at $60 \mathrm{rpm}$. Based on a previous study using the cycling time trial test (Suzuki et al. 2016), exercise conditions were set for evaluating full-power cycling test for about 10-min.

Blood was immediately collected after the 10-min pedaling test, then the participants provided responses to a visual analogue scale (VAS) questionnaire about their perception of physical exertion (muscle fatigue, leg muscle soreness) and subjective conditions (vigor, ease of pedaling, concentration, eyestrain, and blurred vision). The participants subjectively rated their degree of discomfort on a VAS from 0 (no discomfort) to 100 (extreme discomfort) $\mathrm{mm}$ after the 10 -min pedaling test. The VAS was originally developed for measuring pain (Maxwell 1978) and has also been used to assess fatigue (Leung et al. 2004).

No adverse events developed during the study.

\section{Blood analysis}

Plasma was prepared from blood samples by centrifugation at $1000 \times g$ and $4{ }^{\circ} \mathrm{C}$ for $10 \mathrm{~min}$. Plasma $(250 \mu \mathrm{L})$ was mixed with an equal volume of $6 \%$ trichloroacetic acid (for amino 
acid analysis) or $100 \%$ methanol (for NOx analysis), placed on ice for $1 \mathrm{~h}$, separated by centrifugation at $13,000 \times \mathrm{g}$ and $4{ }^{\circ} \mathrm{C}$ for $10 \mathrm{~min}$, and then the supernatant was separated. Free amino acids and NOx in deproteinized plasma were measured using an L-8900 amino acid analyzer (Hitachi High-Technologies Corporation, Tokyo, Japan) and an ENO-20 NOx analyzer (Eicom Corporation., Kyoto, Japan), respectively (Przyborowski et al. 2015).

\section{Statistical analysis}

The number of participants was set to detect the difference in mean values by 0.60 -fold of the standard deviation with an $80 \%$ power at an $\alpha$ level of 0.05 in the paired $t$ test using $\mathrm{R}$ version 3.2.2. Individual power output was calculated as the mean power of the period, whereas the peak pedaling speed was the max pedaling speed of the period. Values are expressed as means or estimated marginal mean values \pm standard error of the mean (SEM). The mean difference of the two paired groups was analyzed by paired test if the normality was hypothesized by Shapiro-Wilk test, otherwise by Wilcoxon's signed rank test. The mean difference of repeated measure data was analyzed by repeated measure ANOVA if the normality was hypothesized by Shapiro-Wilk test, otherwise by generalized linear model (generalized estimating equation procedure). Correlations were analyzed using Pearson's correlation coefficients. All data was statistically analyzed using with SPSS Statistics 22 (IBM Japan, Ltd., Tokyo, Japan). $P$ values $<0.05$ and correlation coefficient $\geq 0.4$ were regarded as significant.

\section{Results}

\section{0-min pedaling tests}

Mean power output was significantly higher in the Cit + Arg group than in the placebo group throughout the 10-min pedaling test $(242 \pm 24$ vs. $231 \pm 21 \mathrm{~W} ; p<0.05$; Fig. 1a) and in the third and fifth quintiles of the 10 -min pedaling test (Fig. 1b).

The peak pedaling speed was not different between the $\mathrm{Cit}+\mathrm{Arg}$ and placebo groups during the test $(99.0 \pm 28.4$ vs. $93.8 \pm 29.3 \mathrm{rpm} ; p=0.297$; Fig. 1 c) nor in any quintile of the test (Fig. 1d).

\section{Plasma NOx on day 7}

The 10-min pedaling test revealed significantly elevated plasma NOx concentrations in both groups $(p<0.05)$. However, the Cit + Arg group showed significantly higher plasma NOx concentration than that in placebo group after the test $(45.2 \pm 2.4$ vs. $37.8 \pm 2.4 \mu \mathrm{M} ; p<0.05)$. The change in NOx concentrations before and after the exercise was significantly higher in the Cit + Arg group than in the placebo group (7.5 \pm 1.3 vs. $3.7 \pm 1.0 \mu \mathrm{M} ; p<0.05)$.

\section{Plasma amino acids on day 7}

The plasma Arg level was significantly higher in the Cit + Arg than in the placebo group before the 10-min pedaling test $(97.0 \pm 5.0$ vs. $81.7 \pm 5.0 \mu \mathrm{M} ; p=0.05$; Fig. $2 \mathrm{c})$. After the test, plasma Cit $(147.4 \pm 7.5$ vs. $32.8 \pm 5.8 \mu \mathrm{M} ; p<0.01)$ and Arg $(158.5 \pm 6.1$ vs. $79.8 \pm 6.1 \mu \mathrm{M} ; p<0.01)$ levels were significantly higher in the $\mathrm{Cit}+\mathrm{Arg}$ group than in the placebo group. A comparison of plasma Cit and Arg levels before and after the exercise showed that plasma Cit levels did not change after the test in the placebo group, whereas plasma Cit $(35.7 \pm 1.4-147.4 \pm 7.5 \mu \mathrm{M} ; p<0.01)$ and Arg $(96.9 \pm 5.0-158.5 \pm 6.1 \mu \mathrm{M} ; p<0.01)$ levels were significantly higher in the Cit + Arg group post-exercise (Fig. 2b, c).

Levels of plasma branched chain amino acids (BCAA; L-valine, L-leucine, and L-isoleucine) were significantly lower after exercise, regardless of ingestion of $\mathrm{Arg}+\mathrm{Cit}$ $(478.7 \pm 13.4$ to $409.6 \pm 10.4 \mu \mathrm{M} ; p<0.01)$ or placebo ( $460.8 \pm 13.4$ to $397.4 \pm 10.4 \mu \mathrm{M} ; p<0.01$ ) (Fig. $2 \mathrm{~d}$ ).

\section{Subjective physical exertion and conditions}

Supplementation with Cit + Arg significantly improved subjective perceptions of "leg muscle soreness" $(5.1 \pm 2.4$ vs. $6.1 \pm 1.7 \mathrm{~cm} ; p<0.05)$ and "ease of pedaling" $(5.8 \pm 1.8$ vs. $6.9 \pm 1.9 ; p<0.05)$ when compared to the placebo immediately post-exercise. "Subjective concentration" $(3.4 \pm 1.8$ vs. $4.3 \pm 1.9 \mathrm{~cm} ; p=0.08)$ also improved, whereas muscular fatigue and other subjective conditions did not differ between Cit + Arg and placebo (Fig. 3).

\section{Correlation between physical performance and NOx concentration}

Power output during the final minute of physical exercise tended to correlate positively with plasma NOx concentration in the $\mathrm{Cit}+\operatorname{Arg}$ group $(R=0.40, p=0.08)$.

\section{Physical parameters}

Height, weight, blood pressure (systolic and diastolic) and heart rate at rest did not significantly differ between the two groups before the exercise on day 7 (data not shown). 

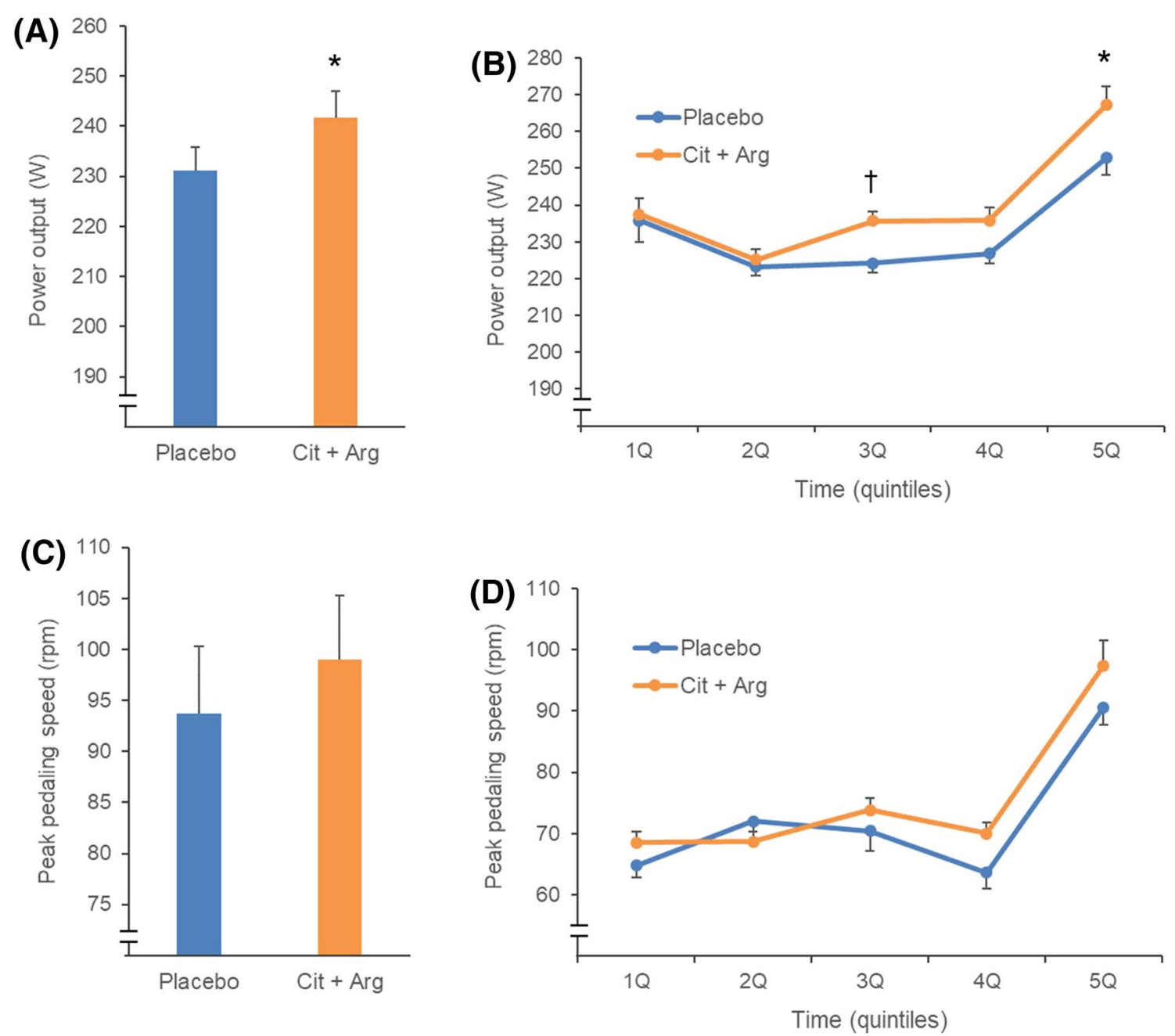

Fig. 1 Results of 10-min pedaling exercise tests. Mean power output during total of $10 \mathrm{~min}$ (a) and the first to fifth quintiles ( 2 min each) (b). Max pedaling speed during total of $10 \mathrm{~min}$ (c) and the first to fifth quintiles ( 2 min each) (d). a, c were analyzed by paired $t$ test,

\section{Discussion}

The ingestion of Cit $+\operatorname{Arg}(1.2 \mathrm{~g} /$ day each) for 7 days significantly increased 10 min cycling performance when compared to a placebo. In the Cit + Arg group, changes in plasma NOx levels before and after exercise and plasma Cit and Arg levels after exercise were significantly higher than in the placebo group. Immediately after the exercise, subjective perceptions of "leg muscle soreness" and "ease of pedaling" were also significantly improved in the Cit + Arg group.

Dietary $\mathrm{NO}_{3}{ }^{-}$-containing supplements, such as beetroot juice, have been reported to exert beneficial effects on exercise performance (Bailey et al. 2009; Lansley et al. 2011b; Cermak et al. 2012a; Wilkerson et al. 2012; Jones 2014). NO could be generated chemically: the ingested $\mathrm{NO}_{3}{ }^{-}$enters the enterosalivary system and and $\mathbf{b}$ and $\mathbf{d}$ were analyzed by generalized estimating equation. Values are means \pm SEM $n=20,{ }^{*} p<0.05,{ }^{\dagger} p<0.01$ indicate a significant difference from placebo

may subsequently be reduced to $\mathrm{NO}_{2}$ by bacterial activity, and $\mathrm{NO}_{2}$ could then be further reduced to $\mathrm{NO}$ (Duncan et al. 1995; Lundberg and Govoni 2004). On the other hand, plasma NO levels derived from Arg-Cit conversion (Schmidt et al. 1988) increase with exercise (Node et al. 1997), and are considered to enhance peripheral circulation and improve exercise performance (Shen et al. 1994; Larsen et al. 2007, 2011; Bailey et al. 2009; Lansley et al. 2011b; Cermak et al. 2012a; Wilkerson et al. 2012; Jones 2014). In fact, voluntary physical activities including running speed and distance are reduced in eNOS knockout mice (Momken et al. 2004). The present study found that $\mathrm{Cit}+$ Arg significantly increased plasma Cit, Arg and NOx levels in association with exercise when compared to a placebo. In addition, plasma NOx concentrations positively correlated with power output in the Cit + Arg group. 

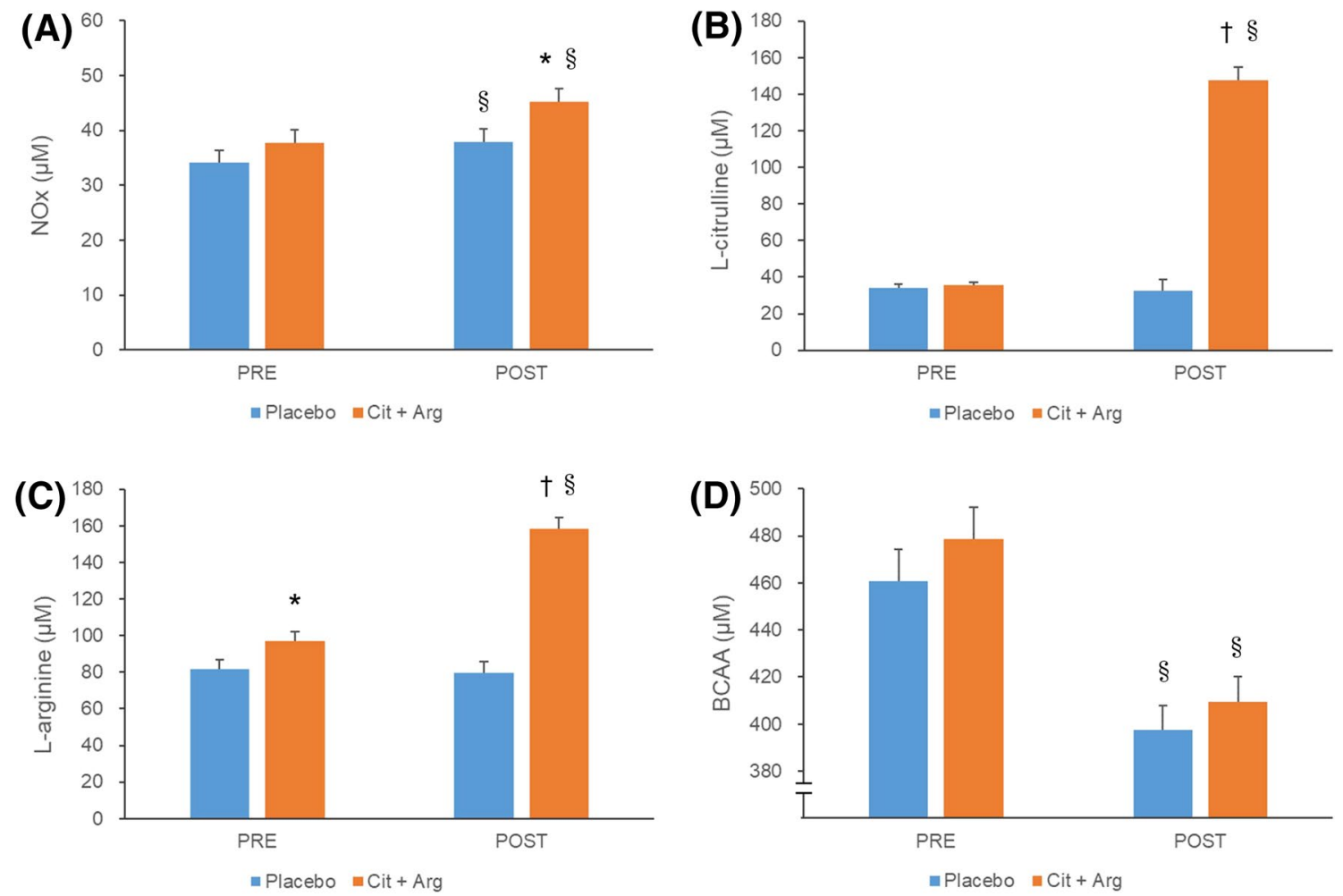

Fig. 2 Plasma a NOx, b L-citrulline, c L-arginine, and d branched chain amino acids (BCAA) concentrations in placebo (blue bars) and $\mathrm{Cit}+\mathrm{Arg}$ (orange bars) groups on day 7. Participants ingested $\mathrm{Cit}+\mathrm{Arg}$ and then participated in 10-min ergometer cycle tests. Plasma NOx and amino acids were analyzed before supplement ingestion (PRE) and after exercise (POST). a, c, d Were analyzed by

repeated measure ANOVA, and $\mathbf{b}$ was analyzed by generalized estimating equation. Height of bars and error bars represent mean values and SEM, respectively. Symbols above bars represent statistical significance as follows: ${ }^{*} p<0.05$ and ${ }^{\dagger} p<0.01$, between groups vs. placebo group, and ${ }^{\S} p<0.01$, within groups vs. PRE

Fig. 3 Subjective feelings immediately after exercise. Length represents perceived degree of discomfort on visual analog scale from 0 (no discomfort to 100 (extreme discomfort) $\mathrm{mm}$, with higher values representing worsening discomfort. Mean difference was analyzed by Wilcoxon's signed rank test, except for "Tension of the leg" and "Concentration" by paired $t$ test. Values are means \pm SEM, $n=20, * p<0.05$; significantly different from placebo

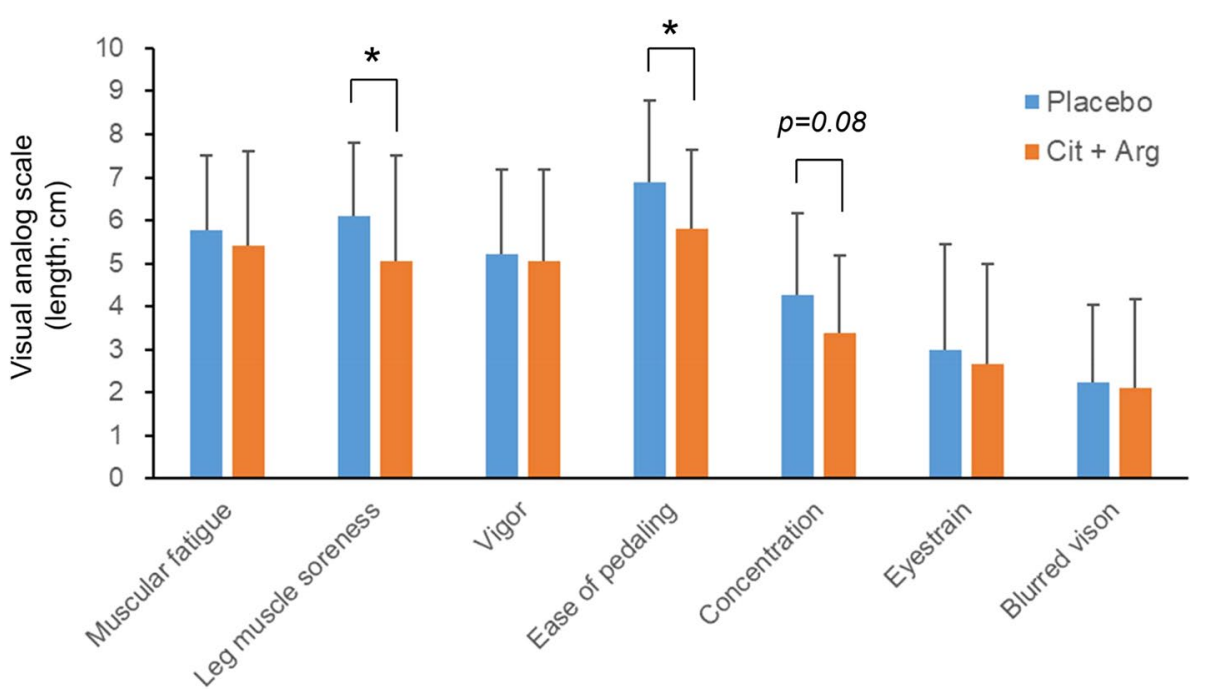

Therefore, the elevated Cit and Arg values could enhance exercise-induced $\mathrm{NO}$ and improve cycling performance.

Ingested Arg is susceptible to arginase degradation to ornithine in the gastrointestinal tract and liver (Fig. 4) and thus it cannot effectively elevate or maintain plasma levels of Arg. We previously showed that plasma Arg levels returned to the baseline within $4 \mathrm{~h}$ after the ingestion of $2.0 \mathrm{~g}$ of Arg (Suzuki et al. 2017). This could account for why high doses 


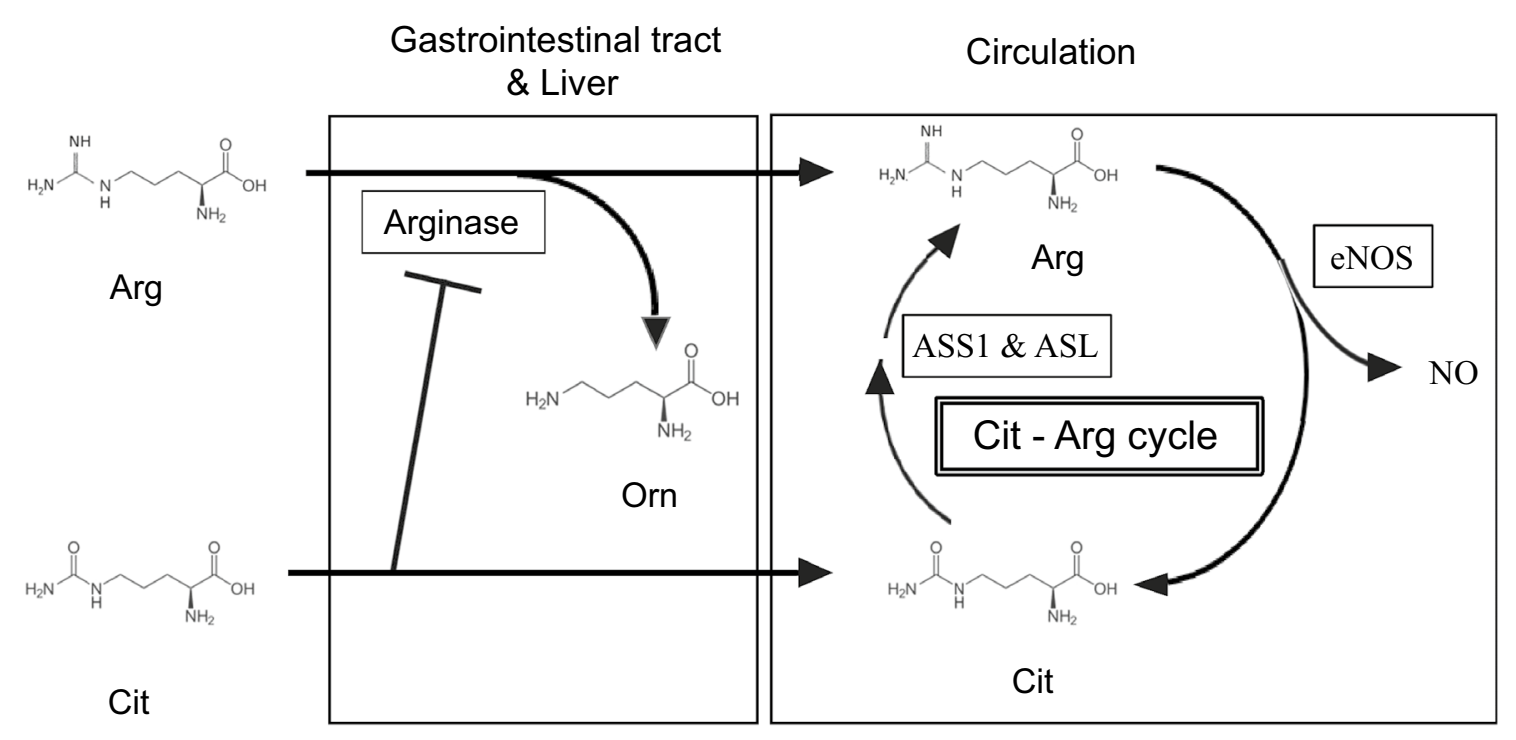

Fig. 4 Metabolic pathways of L-arginine (Arg) and L-citrulline (Cit). Ingested Arg is mainly degraded in gastrointestinal tract and liver by arginase which is inhibited by Cit. Therefore, co-ingested Cit protects Arg and elevates circulating Arg concentrations. Endothelial nitric

of 6-14.2 g/day are needed to improve exercise function in healthy persons (Campbell et al. 2006; Fricke et al. 2008; Koppo et al. 2009).

Cit is not metabolized in the small intestine, and it inhibits the degradation of Arg by arginase. Thus, oral Cit + Arg synergistically increases plasma Arg levels in mice and humans compared with its ingestion of either alone (Morita et al. 2014; Suzuki et al. 2017). Circulating Arg is converted to NO and Cit by eNOS. Cit then is recycled to Arg by ASS1 and ASL in an efficient cycle of NO production in the circulatory system (Fig. 4) (Curis et al. 2005). In this study, plasma Cit levels increased significantly after Cit + Arg intake. This may provide an explanation, in that arginase suppression, mediated by Cit, probably contributes to a marked increase in Arg availability.

However, our previous study did not show elevated Arg levels on the day after ingesting Cit and Arg (1.0 g/ day of each) for 7 days (Suzuki et al. 2017). The present study found that orally ingested Cit + Arg (1.2 g/day each for 6 days) significantly increased plasma Arg levels when compared to a placebo before Cit + Arg ingestion on day 7 . The participants did not ingest the supplement after 15:00 on day 6 , and blood was collected $>17 \mathrm{~h}$ after the final ingestion. The combination of $\mathrm{Cit}+\operatorname{Arg}(1.2 \mathrm{~g} / \mathrm{day}$ each) therefore increased and maintained plasma Arg concentrations.

The discrepancy in plasma Arg concentrations between the previous (Suzuki et al. 2017) and the present studies could be due to differences in the doses and the background characteristics of the participants, who consisted of obese men in the previous study (Suzuki et al. 2017) and collegiate oxide (NO) synthase (eNOS) converts Arg to NO and Cit in circulation. Thereafter, Cit is then recycled to Arg by argininosuccinate synthase (ASS1) and argininosuccinate lyase (ASL). This Cit-Arg cycle enables effective $\mathrm{NO}$ production

athletes in the present study. Arginase activity is higher in patients with hypertension and diabetes, and this decreases Arg bioavailability (Shatanawi et al. 2011; Pernow and Jung 2013). Another study has shown that the ingestion of $2.4 \mathrm{~g} /$ day of Cit for 7 days increased plasma Arg levels on day 8 in men who regularly exercised (Suzuki et al. 2016). Another possible explanation for the immediate and synergistic increase in plasma Arg and NO by oral Cit + Arg ingestion might be the inhibition of arginase by Cit (Romero et al. 2008). Cit suppresses arginase activity in vitro and in vivo through its powerful allosteric inhibitory action (Shearer et al. 1997; Romero et al. 2008; El-Bassossy et al. 2012). The Arg transporters rBAT/b0, AT, and CAT- 1 and the Cit transporter SN-1 are simultaneously expressed in intestinal epithelial and vascular endothelial cells (Closs et al. 2004; Broer 2008; Romero et al. 2008). Thus, Cit might be absorbed together with Arg when combined Cit and Arg are ingested, inhibiting arginase in intestinal and endothelial cells, and thus increasing Arg concentrations and NO production. The elevated plasma Cit concentration in the present study could account for this mechanism to increase plasma Arg and NO concentration. Therefore, Cit + Arg, at relatively low dosages, could increase Arg levels, especially in athletes.

Some studies have shown that a single administration of oral Cit does not improve exercise performance (Hickner et al. 2006; Cutrufello et al. 2015), whereas 6-7 days of Cit supplementation improves exercise tolerance (Bailey et al. 2015; Suzuki et al. 2016). The present study showed performance in the 10-min pedaling test to be enhanced after 
7 days of Cit + Arg supplementation. These findings suggest that, to improve exercise performance, Cit and Arg should be continuously ingested. Arginine and Cit, both individually and in combination, elevate endothelial NO synthase levels and decrease those of arginase in human vascular endothelial cells (HUVECs) after 3 days of incubation (Tsuboi et al. 2018). The duration of Arg and Cit ingestion might have altered the endothelial capacity for NO production.

Other factors require further exploration. Two reports have indicated that the oral intake of Cit significantly decreases plasma BCAA levels (Sureda et al. 2010; Suzuki et al. 2016), but the present study found no significant differences in these levels. The relationship between Cit and BCAA remains to be determined. L-citrulline-malate and watermelon juice, which is rich in Cit, can alleviate the delayed onset of muscle soreness (Pérez-Guisado and Jakeman 2010; Tarazona-Díaz et al. 2013). The present study did not measure muscle pain on the day after the exercise test or beyond, but subjective perceptions of "leg muscle soreness" and "ease of pedaling" immediately after the exercise were improved. Therefore, Cit or the resulting NO could influence subjective perceptions, and the mechanism behind this requires further investigation.

The present study had several limitations. The kinetics of plasma amino acids and NOx concentrations were not measured. Notably, increases in plasma NOx levels induced by the exercise were much lower than those measured in the previous literature (Jungersten et al. 1997; Franco et al. 2001; Suzuki et al. 2016). The discrepancy could be due to the timing of blood collection. We collected post-exercise blood immediately after the exercise; nevertheless, the NOx concentration induced by high-intensity exercise is reported to be elevated for up to $24 \mathrm{~h}$ after exercise (Güzel et al. 2007), which could suggest that the time-point for collecting the blood in our study was not suitable for detecting the peak in nitrate levels. Because a significant difference was observed in post-exercise NOx concentrations, the difference could have been more apparent had we checked later, preferably up to $48 \mathrm{~h}$ after the exercise. Therefore, a closer study of the timing of supplementation and exercise is needed. This study indicated $1.2 \mathrm{~g} /$ day of $\mathrm{Cit}$ is the minimum dose needed to evaluate a potential beneficial effect on exercise performance, but that this is combined with the same amount of Arg. The dose-response effect of Cit + Arg needs to be more precisely determined. The duration of the supplementation should also be assessed, because we tested a duration of 7 days in vivo, whereas NO synthase and arginase levels are up-regulated in HUVECs in vitro within 3 days (Tsuboi et al. 2018). Furthermore, our participants comprised 20 male collegiate soccer players. Thus, more participants with various backgrounds, including females, sedentary persons and recreational athletes, should be evaluated to give the conclusion a broader relevance.

\section{Conclusion}

Oral ingestion of Cit and Arg at doses of $1.2 \mathrm{~g} /$ day each for 7 days improved exercise performance in a 10-min pedaling test and the participants' subjective perceptions of physical exertion. The increased NO production induced by elevated plasma Cit and Arg levels could account for this effect.

Authors' contributions IS: served as the study coordinator and was involved in participant recruitment, testing, laboratory analyses, and writing of the draft manuscript. KS: contributed to acquisition of participants' consent and instruction of participants. TH: advised on study design and helped to carry out the study. TK: contributed to instruction of participants and laboratory analyses. JY: contributed to instruction of participants and laboratory analyses. TS, MM, and AN: contributed to study design and manuscript preparation. YS: was the principal investigator developing the experimental design. He was also involved in training and mentoring for laboratory analyses and supervised manuscript preparation. All authors read and approved the final manuscript.

\section{Compliance with ethical standards}

Conflict of interest This study was funded by Kyowa Hakko Bio Co. Ltd. Takashi Suzuki, Masahiko Morita, and Akihito Nishimura are employees of Kyowa Hakko Bio Co. Ltd. None of these authors play any roles that might have affected the conclusions. The other co-authors have no conflict of interests to declare.

OpenAccess This article is distributed under the terms of the Creative Commons Attribution 4.0 International License (http://creativeco mmons.org/licenses/by/4.0/), which permits unrestricted use, distribution, and reproduction in any medium, provided you give appropriate credit to the original author(s) and the source, provide a link to the Creative Commons license, and indicate if changes were made.

\section{References}

Albrecht EWJA, Stegeman CA, Heeringa P et al (2003) Protective role of endothelial nitric oxide synthase. J Pathol 199:8-17. https://doi.org/10.1002/path.1250

Bailey SJ, Winyard P, Vanhatalo A et al (2009) Dietary nitrate supplementation reduces the $\mathrm{O} 2$ cost of low-intensity exercise and enhances tolerance to high-intensity exercise in humans. J Appl Physiol 107:1144-1155. https://doi.org/10.1152/jappl physiol.00722.2009-Pharmacological

Bailey SJ, Fulford J, Vanhatalo A et al (2010) Dietary nitrate supplementation enhances muscle contractile efficiency during kneeextensor exercise in humans. J Appl Physiol 109:135-148. https ://doi.org/10.1152/japplphysiol.00046.2010

Bailey SJ, Blackwell JR, Lord T et al (2015) 1-citrulline supplementation improves $\mathrm{O}_{2}$ uptake kinetics and high-intensity exercise performance in humans. J Appl Physiol 119:385-395. https:// doi.org/10.1152/japplphysiol.00192.2014

Bescós R, Sureda A, Tur JA, Pons A (2012) The effect of nitric-oxiderelated supplements on human performance. Sports Med 42:99_ 117. https://doi.org/10.2165/11596860-000000000-00000 
Bode-Boger SM, Muke J, Surdacki A et al (2003) Oral l-arginine improves endothelial function in healthy individuals older than 70 years. Vasc Med 8:77-81. https://doi.org/10.1191/13588 $63 \times 03$ vm 474 oa

Boorsma RK, Whitefield J, Spriet LL (2014) Beetroot juice supplementation does not improve performance of elite $1500-\mathrm{m}$ runners. Med Sci Sport Exerc 46:2326-2334. https://doi. org/10.1249/MSS.0000000000000364

Broer S (2008) Amino acid transport across mammallian intestinal and renal epithelia. Physiol Rev 88:249-286. https://doi. org/10.1152/physrev.00018.2006

Campbell B, Roberts M, Kerksick C et al (2006) Pharmacokinetics, safety, and effects on exercise performance of L-arginine $\alpha$-ketoglutarate in trained adult men. Nutrition 22:872-881. https ://doi.org/10.1016/j.nut.2006.06.003

Castillo L, Chapman TE, Sanchez M et al (1993a) Plasma arginine and citrulline kinetics in adults given adequate and arginine-free diets. Proc Natl Acad Sci U S A 90:7749-7753. https://doi.org/10.1073/ pnas.90.16.7749

Castillo L, DeRojas TC, Chapman TE et al (1993b) Splanchnic metabolism of dietary arginine in relation to nitric oxide synthesis in normal adult man. Proc Natl Acad Sci U S A 90:193-197. https ://doi.org/10.1073/pnas.90.1.193

Cermak NM, Gibala MJ, Van Loon LJC (2012a) Nitrate supplementation's improvement of $10-\mathrm{km}$ time-trial performance in trained cyclists. Int J Sport Nutr Exerc Metab 22:64-71. https://doi. org/10.1123/ijsnem.22.1.64

Cermak NM, Res P, Stinkens R et al (2012b) No improvement in endurance performance after a single dose of beetroot juice. Int $\mathrm{J}$ Sport Nutr Exerc Metab 22:470-478

Chen J, Wollman Y, Chernichovsky T et al (1999) Effect of oral administration of high-dose nitric oxide donor 1-arginine in men with organic erectile dysfunction: results of a double-blind, randomized, placebo-controlled study. BJU Int 83:269-273. https:// doi.org/10.1046/j.1464-410X.1999.00906.x

Closs EI, Simon A, Ve N, Rotmann A (2004) Plasma membrane transporters for arginine. J Nutr 134:2752S-2759S

Curis E, Nicolis I, Moinard C et al (2005) Almost all about citrulline in mammals. Amino Acids 29:177-205. https://doi.org/10.1007/ s00726-005-0235-4

Cutrufello PT, Gadomski SJ, Zavorsky GS (2015) The effect of L-citrulline and watermelon juice supplementation on anaerobic and aerobic exercise performance. J Sports Sci 33:1459-1466. https ://doi.org/10.1080/02640414.2014.990495

Duncan C, Dougall H, Johnston P et al (1995) Chemical generation of nitric oxide in the mouth from the enterosalivary circulation of dietary nitrate. Nat Med 1:546-551

El-Bassossy HM, El-Fawal R, Fahmy A (2012) Arginase inhibition alleviates hypertension associated with diabetes: effect on endothelial dependent relaxation and NO production. Vasc Pharmacol 57:194-200. https://doi.org/10.1016/j.vph.2012.01.001

Franco L, Doria D, Mattiucci F (2001) Effect of acute exercise on plasma nitric oxide level in humans. Med Princ Pract 10:106-109. https://doi.org/10.1159/000050351

Fricke O, Baecker N, Heer M et al (2008) The effect of 1-arginine administration on muscle force and power in postmenopausal women. Clin Physiol Funct Imaging 28:307-311. https://doi. org/10.1111/j.1475-097X.2008.00809.x

Güzel NA, Hazar S, Erbas D (2007) Effects of different resistance exercise protocols on nitric oxide, lipid peroxidation and creatine kinase activity in sedentary males. J Sport Sci Med 6:417-422. https://doi.org/10.1111/j.1399-5618.2005.00251.x

Hickner RC, Tanner CJ, Evans CA et al (2006) 1-citrulline reduces time to exhaustion and insulin response to a graded exercise test. Med Sci Sports Exerc 38:660-666. https://doi.org/10.1249/01. mss.0000210197.02576.da
Jones AM (2014) Dietary nitrate supplementation and exercise performance. Sport Med 44:S35-S45. https://doi.org/10.1007/s4027 9-014-0149-y

Jones AM, Vanhatalo A, Bailey SJ (2013) Influence of dietary nitrate supplementation on exercise tolerance and performance. In: Nestle Nutrition Institute workshop series. pp 27-40

Jungersten L, Ambring A, Wall B, Wennmalm A (1997) Both physical fitness and acute exercise regulate nitric oxide formation in healthy humans. J Appl Physiol 82:760-764. https://doi. org/10.1152/jappl.1997.82.3.760

Koppo K, Taes YE, Pottier A et al (2009) Dietary arginine supplementation speeds pulmonary VO2 kinetics during cycle exercise. Med Sci Sports Exerc 41:1626-1632. https://doi.org/10.1249/ MSS.0b013e31819d81b6

Lansley KE, Winyard PG, Bailey SJ et al (2011a) Acute dietary nitrate supplementation improves cycling time trial performance. Med Sci Sports Exerc 43:1125-1131. https://doi.org/10.1249/ MSS.0b013e31821597b4

Lansley KE, Winyard PG, Fulford J et al (2011b) Dietary nitrate supplementation reduces the $\mathrm{O} 2$ cost of walking and running: a placebo-controlled study. J Appl Physiol 110:591. https://doi. org/10.1152/japplphysiol.01070.2010

Larsen FJ, Weitzberg E, Lundberg JO, Ekblom B (2007) Effects of dietary nitrate on oxygen cost during exercise. Acta Physiol 191:59-66. https://doi.org/10.1111/j.1748-1716.2007.01713.x

Larsen FJ, Schiffer TA, Borniquel S et al (2011) Dietary inorganic nitrate improves mitochondrial efficiency in humans. Cell Metab 13:149-159. https://doi.org/10.1016/j.cmet.2011.01.004

Leung AWS, Chan CCH, Lee AHS, Lam KWH (2004) Visual analogue scale correlates of musculoskeletal fatigue. Percept Mot Skills 99:235-246. https://doi.org/10.2466/pms.99.1.235-246

Lin CC, Tsai WC, Chen JY et al (2008) Supplements of 1-arginine attenuate the effects of high-fat meal on endothelial function and oxidative stress. Int J Cardiol 127:337-341. https://doi. org/10.1016/j.ijcard.2007.06.013

Lundberg JO, Govoni M (2004) Inorganic nitrate is a possible source for systemic generation of nitric oxide. Free Radic Biol Med 37:395-400. https://doi.org/10.1016/j.freeradbiomed.2004.04.027

Maxwell C (1978) Sensitivity and accuracy of the visual analogue scale: a psycho-physical classroom experiment. Br J Clin Pharmacol 6:15-24. https://doi.org/10.1111/j.1365-2125.1978.tb01676.x

Miyashita M, Mutoh Y, Yoshioka N, Sadamoto T (1985) PWC75\%HRmax: a measure of aerobic work capacity. Sports Med 2:159-164

Momken I, Lechêne P, Ventura-Clapier R, Veksler V (2004) Voluntary physical activity alterations in endothelial nitric oxide synthase knockout mice. Am J Physiol Circ Physiol 287:H914-H920. https ://doi.org/10.1152/ajpheart.00651.2003

Morita M, Hayashi T, Ochiai M et al (2014) Oral supplementation with a combination of 1-citrulline and 1-arginine rapidly increases plasma 1-arginine concentration and enhances NO bioavailability. Biochem Biophys Res Commun 454:53-57. https://doi. org/10.1016/j.bbrc.2014.10.029

Nisoli E, Clementi E, Paolucci C et al (2003) Mitochondrial biogenesis in mammals: the role of endogenous nitric oxide. Science 299:896-899. https://doi.org/10.1126/science.1079368

Node K, Kitakaze M, Sato H et al (1997) Effect of acute dynamic exercise on circulating plasma nitric oxide level and correlation to norepinephrine release in normal subjects. Am J Cardiol 79:526-528. https://doi.org/10.1016/S0002-9149(96)00804-1

Ochiai M, Hayashi T, Morita M et al (2012) Short-term effects of L-citrulline supplementation on arterial stiffness in middle-aged men. Int J Cardiol 155:257-261. https://doi.org/10.1016/j.ijcar d.2010.10.004

Pérez-Guisado J, Jakeman PM (2010) Citrulline malate enhances athletic anaerobic performance and relieves muscle soreness. 
J Strength Cond Res 24:1215-1222. https://doi.org/10.1519/ JSC.0b013e3181cb28e0

Pernow J, Jung C (2013) Arginase as a potential target in the treatment of cardiovascular disease: reversal of arginine steal? Cardiovasc Res 98:334-343. https://doi.org/10.1093/cvr/cvt036

Petróczi A, Naughton DP (2010) Potentially fatal new trend in performance enhancement: a cautionary note on nitrite. J Int Soc Sports Nutr 7:25. https://doi.org/10.1186/1550-2783-7-25

Przyborowski K, Wojewoda M, Sitek B et al (2015) Effects of 1-methylnicotinamide (MNA) on exercise capacity and endothelial response in diabetic mice. PLoS One 10:1-15. https://doi. org/10.1371/journal.pone.0130908

Quijano C, Trujillo M, Castro L, Trostchansky A (2016) Interplay between oxidant species and energy metabolism. Redox Biol 8:28-42. https://doi.org/10.1016/j.redox.2015.11.010

Romero MJ, Platt DH, Tawfik HE et al (2008) Diabetes-induced coronary vascular dysfunction involves increased arginase activity. Circ Res 102:95-102. https://doi.org/10.1161/CIRCRESAHA .107 .155028

Schmidt HHHW, Nau H, Wittfoht W et al (1988) Arginine is a physiological precursor of endothelium-derived nitric oxide. Eur J Pharmacol 154:213-216. https://doi.org/10.1016/0014-2999(88)90101 $-\mathrm{X}$

Schwedhelm E, Maas R, Freese R et al (2008) Pharmacokinetic and pharmacodynamic properties of oral L-citrulline and L-arginine: impact on nitric oxide metabolism. Br J Clin Pharmacol 65:51-59. https://doi.org/10.1111/j.1365-2125.2007.02990.x

Shatanawi A, Romero M, Iddings J et al (2011) Angiotensin II-induced vascular endothelial dysfunction through RhoA/Rho kinase/p38 mitogen-activated protein kinase/arginase pathway. Am J Physiol Cell Physiol 300:C1181-C1192. https://doi.org/10.1152/ajpce 11.00328 .2010

Shearer JD, Richards JR, Mills CD, Caldwell MD (1997) Differential regulation of macrophage arginine metabolism: a proposed role in wound healing. Am J Physiol 272:E181-E190

Shen W, Xu X, Ochoa M et al (1994) Role of nitric oxide in the regulation of oxygen consumption in conscious dogs. Circ Res 75:10861095. https://doi.org/10.1161/01.RES.75.6.1086

Siasos G, Tousoulis D, Vlachopoulos C et al (2008) Short-term treatment with l-arginine prevents the smoking-induced impairment of endothelial function and vascular elastic properties in young individuals. Int J Cardiol 126:394-399. https://doi.org/10.1016/j. ijcard.2007.04.057

Sureda A, Córdova A, Ferrer MD et al (2010)) 1-citrulline-malate influence over branched chain amino acid utilization during exercise. Eur J Appl Physiol 110:341-351. https://doi.org/10.1007/s0042 $1-010-1509-4$
Suzuki T, Morita M, Kobayashi Y, Kamimura A (2016) Oral 1-citrulline supplementation enhances cycling time trial performance in healthy trained men: double-blind randomized placebo-controlled 2-way crossover study. J Int Soc Sports Nutr 13:6. https://doi. org/10.1186/s12970-016-0117-z

Suzuki T, Morita M, Hayashi T, Kamimura A (2017) The effects on plasma L-arginine levels of combined oral L-citrulline and L-arginine supplementation in healthy males. Biosci Biotechnol Biochem 81:372-375. https://doi.org/10.1080/09168451.2016.12300 07

Tarazona-Díaz MP, Alacid F, Carrasco M et al (2013) Watermelon juice: potential functional drink for sore muscle relief in athletes. J Agric Food Chem 61:7522-7528. https://doi.org/10.1021/jf400 $964 \mathrm{r}$

Tschakovsky ME, Joyner MJ (2008) Nitric oxide and muscle blood flow in exercise. Appl Physiol Nutr Metab 33:151-160. https:// doi.org/10.1139/H07-148

Tsuboi T, Maeda M, Hayashi T (2018) Administration of 1-arginine plus 1-citrulline or 1-citrulline alone successfully retarded endothelial senescence. PLoS One 13:e0192252. https://doi.org/10.1371/ journal.pone.0192252

Van De Poll MCG, Soeters PB, Deutz NEP et al (2004) Renal metabolism of amino acids: its role in interorgan amino acid exchange. Am J Clin Nutr 79:185-197. https://doi.org/10.1093/ajcn/79.2.185

Van De Poll MCG, Siroen MPC, Van Leeuwen PAM et al (2007) Interorgan amino acid exchange in humans: consequences for arginine and citrulline metabolism. Am J Clin Nutr 85:167-172

Wilkerson DP, Hayward GM, Bailey SJ et al (2012) Influence of acute dietary nitrate supplementation on 50 mile time trial performance in well-trained cyclists. Eur J Appl Physiol 112:4127-4134. https ://doi.org/10.1007/s00421-012-2397-6

Windmueller HG, Spaeth AE (1981) Source and fate of circulating citrulline. Am J Physiol 241:E473-E480

Wu G (1998) Intestinal mucosal amino acid catabolism. J Nutr 128:1249-1252

Yabuki Y, Shioda N, Yamamoto Y et al (2013) Oral 1-citrulline administration improves memory deficits following transient brain ischemia through cerebrovascular protection. Brain Res 1520:157-167. https://doi.org/10.1016/j.brainres.2013.05.011

Publisher's Note Springer Nature remains neutral with regard to jurisdictional claims in published maps and institutional affiliations. 